\title{
Geographical patterns of karyotype polymorphism in Italian populations of Ornithogalum montanum (Liliaceae)
}

\author{
Guido Barbujani* and \\ Massimo Pigliucci†
}

\author{
* Istituto di Zoologia, Universita' di Ferrara, \\ via L. Borsari, 46 I-44100 Ferrara, Italy. \\ $\dagger$ Dipartimento di Biologia Vegetale, \\ Universita' di Roma "La Sapienza”, \\ Piazzale A. Moro, 7 1-00100 Roma, Italy.
}

The geographical patterns of quinacrine banding polymorphism at 20 sites, and of numbers of accessory (B) chromosomes, were studied in nine Italian populations of Ornithogalum montanum Cyr. ex Ten. (Liliaceae). Eight banding sites appear monomorphic. The standardized gene frequency variance, $F_{\text {st }}$, is heterogeneous among the remaining 12 sites; variation is greatest for four polymorphisms whose frequency is correlated with the winter temperature of the localities studied. A strong negative association is apparent between numbers of B chromosomes and $Q$ bands. Spatial autocorrelation shows three distinct modes of geographical variation: (i) random distributions; (ii) patterns with positive short-range autocorrelation; (iii) patterns with negative intermediate-range autocorrelation. Some microevolutionary implications of these findings are discussed.

\section{INTRODUCTION}

The study of geographical variation of heritable traits may give us insight into the phenomena that lead to population differentiation. Random processes, such as genetic drift and founder effect, determine patchy distributions of gene frequencies (Rohlf and Schnell, 1971; Sokal and Wartenberg, 1983). Also differential selection results in genetic divergence of populations, and it tends to bring about non-random, often clinal, spatial patterns (Endler, 1977). Conversely, gene flow within the area studied and stabilizing selection cause gene frequencies to approach the same values in various populations. The geographical distribution of allele frequencies results from interaction among these processes. It is possible to draw inferences on such processes based on the patterns observed (Sokal and Oden, 1978b; Felsenstein, 1982).

The majority of recent studies have dealt with description and interpretation of protein polymorphisms, especially in animals, which represent over 90 per cent of the species reviewed by Nevo et al. (1984) in their extensive analysis of genetic diversity. Populations of plants have been studied in lesser detail, but it is clear that they are highly variable. The reasons for that include the possibility of self-fertilization, limits to dispersal, small breeding size of the isolates, and tight association of the individuals to the environment where they develop (Bradshaw, 1972; Sultan, 1987). From the standpoint of geographic analysis, this means that in plants gene flow will seldom blur gene frequency patterns caused by selection, nor will it tend to create gradients among differentiated isolates. Rather, because of the small size of the isolates, the impact of random genetic drift on the distributions of allele frequencies may be substantial.

Ornithogalum montanum Cyr. ex Ten. (Liliaceae) is a perennial bulbous plant occurring in the Middle East and the Mediterranean area. Its centre of radiation is probably Turkey (Cullen and Ratter, 1967), where O. montanum is sympatric and partially crossing with $O$. lanceolatum; its chromosome number varies from $n=8$ to $n=11$. On the contrary, Italian specimens show a stable karyotype $(n=9$ : Tornadore, 1986), highly variable heterochromatization patterns (from 0 to 26 Q-bands per karyotype), and up to eight accessory (B) chromosomes (Capineri et al., 1979). Bchromosomes are non-essential supernumeraries, not homologous with members of the basic chromosome complement, which are transmitted in a non-Mendelian fashion (Jones and Rees, 1982; Jones, 1985). O. lanceolatum is not present in Italy.

Spatial analysis is informative on the processes maintaining genetic polymorphism, especially in the presence of high levels of variation, and 


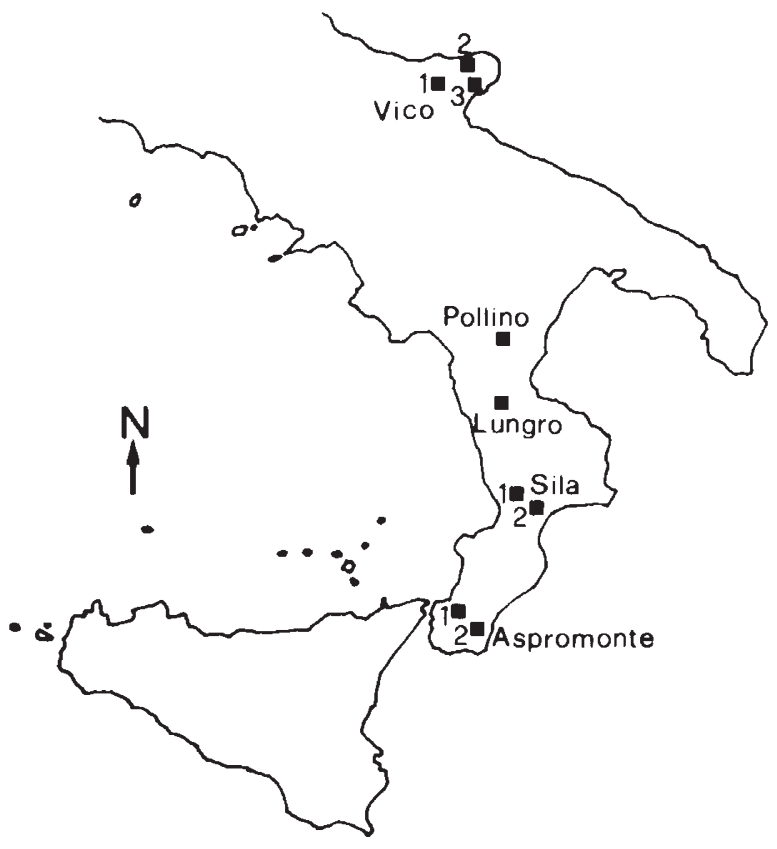

Figure 1 A map of the sampling localities.

isolation of population units. The karyotype of Italian $O$. montanum represents, therefore, an appealing system for microevolutionary inferences. In this study, chromosome banding sites are treated as biallelic loci, which can carry or not carry the Q-band; these alternatives will be here indicated by a "+" for presence of a band, a "-" for its absence. Variation of frequencies of these bands will be summarized by Wright's (1965) $F_{\text {st }}$, the standardized variance of gene frequency. Further, the geographical patterns will be described by means of correlation and spatial autocorrelation coefficients. Inferences on the origin and maintenance of karyotype polymorphism will eventually be possible on the basis of these statistics (Sokal and Oden, 1978b; Sokal, 1979, 1986). In particular, five questions are addressed. (1) Is geographic variation of karyotypes random? If not, (2) do the spatial patterns reveal a continuum of variation and the effects of isolation by distance, or do they rather suggest that the Italian population is subdivided into independent entities? (3) Are clines present, thus supporting the view that karyotype differentiation has been established in response to gradual ecological variation? (4) Does any evidence suggest that the number of B chromosomes is somehow regulated, or, alternatively, is this number determined just by a simple accumulation mechanism (Jones, 1985)? Finally, (5) which microevolutionary process, or combination of processes, is most likely to account for the observed karyotype diversity?

\section{MATERIALS AND METHODS}

\section{The data set}

A total of 477 specimens was collected in nine localities of Southern Italy (fig. 1). At each locality,

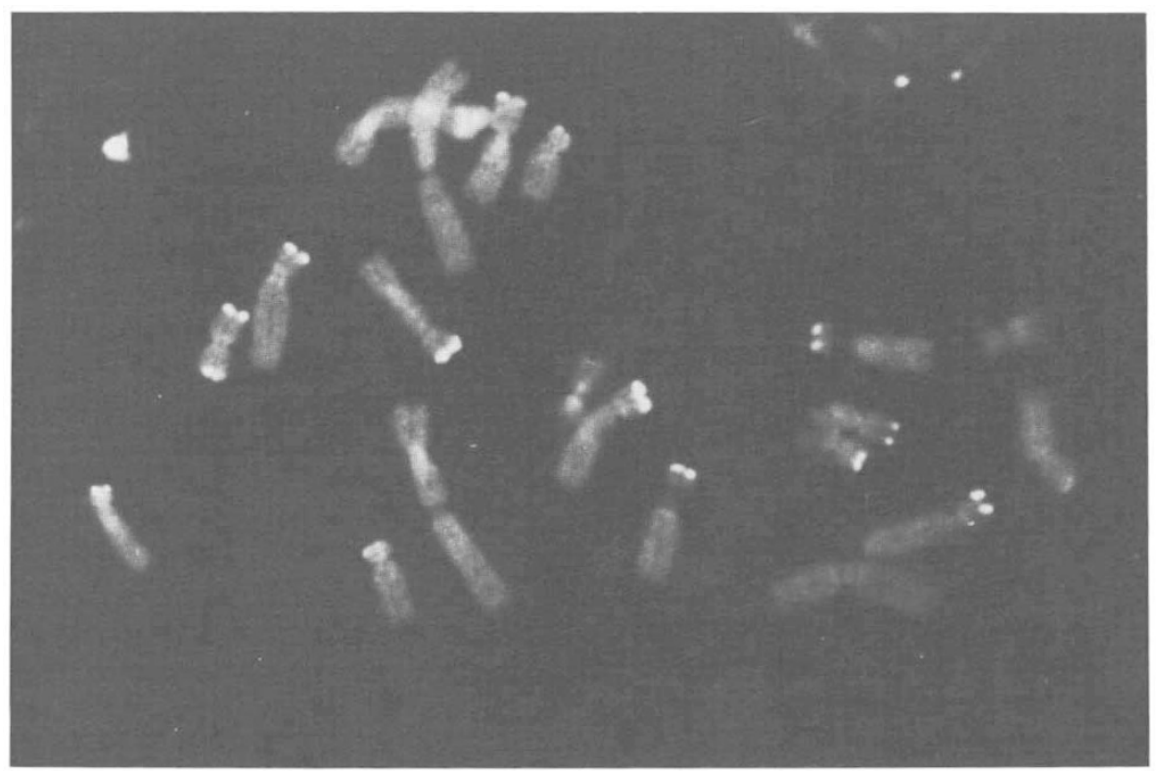

Figure 2 Q-banded metaphase plate of Ornithogalum montanum. Chromosome number of this individual is $2 n=18+2 \mathrm{~B}$. 
a square area of variable extension was delimited and sampled. Each specimen studied was separated by at least 2 metres from its nearest collected neighbour, which rules out repeated sampling of the same karyotype in individuals resulting from asexual reproduction of the same plant.

Karyotypes were reconstructed by means of Feulgen and Quinacrine-banding standard techniques applied on root squashes (fig. 2) (Capineri et al., 1979). All nine chromosomes are metacentric or submetacentric. Q-bands are located in the telomeric section of the chromosome arms, or close to the centromere. Therefore, there are 36 potential banding sites in the karyotype (fig. 3). Chromosome number 1 never shows any bands. Numbers 4 and 5 are morphologically identical and number 8 is perfectly metacentric; as a consequence, their bands could not be identified unambiguously, and the analysis was restricted to the remaining 20 loci, 12 of which were polymorphic. See fig. 3 for the conventional names of these markers.

For each population studied, an array of 14 biological variables was calculated, i.e., the local frequencies of the bands at the loci $\mathrm{B} 2$ to $\mathrm{A} 9$, plus

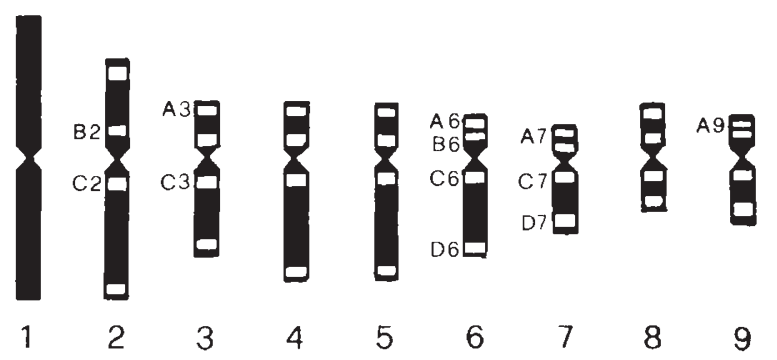

Figure 3 Q-banded idiogram of Ornithogalum montanum. Banding sites that are polymorphic in Italy are indicated by a conventional name. average numbers of $\mathrm{B}$-chromosomes $(\mathrm{NoBC})$ and Q-bands (NoQB). No intra-individual variation of the banding pattern or of the B-chromosome number was observed. In addition, seven environmental measures were available (Anonimous, 1983): latitude (LA), longitude (LO), elevation (EL), average annual rainfall (RF), average temperatures in January (WT) and July (ST), difference in average temperatures July-January $(\Delta \mathrm{T})$ (table 1$)$.

\section{Heterogeneity of $F_{s t}$}

The maximum variance of a gene frequency in a subdivided population is $\bar{q}(1-\bar{q})$, where $\bar{q}$ is the average allele frequency over all subdivisions. The ratio of the observed variance, $s^{2}$, to this theoretical value is $F_{\mathrm{st}}$ (Wright, 1965, 1969). Genetic drift and gene flow must affect all genes equally, and thus the amount of variance actually displayed is expected to be the same at all neutral loci. Differential selection should result in high $F_{\mathrm{st}}$ values at the loci affected; stabilizing selection should have the opposite effect (Cavalli-Sforza, 1966).

The possibility of employing the heterogeneity of $F_{\mathrm{st}}$ values as a test of selective neutrality has long been a matter of debate (Lewontin and Krakauer, 1973, 1975; Nei et al., 1977; Felsenstein, 1982). In the present study, a nonparametric approach was chosen (Barbujani, 1985). Correlation coefficients were calculated between the 12 allele frequencies and the seven environmental variables. The following procedure was then repeated for each of the latter variables: (1) the loci studied were classified into two groups, one including the loci whose allele frequencies correlated significantly with the variable considered,

Table 1 Some characteristics of the populations studied and of their habitats

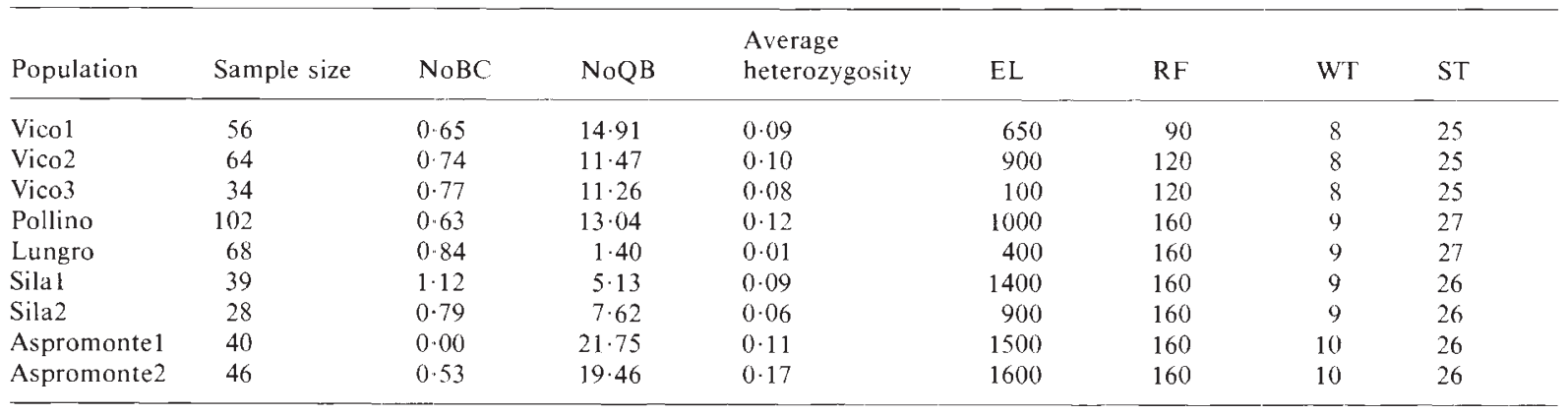

Total

477

NoBC: Average number of B chromosomes; NoQB: Average number of Q bands; EL: Elevation (m); RF: Rainfall (cm); WT: Average January temperature (centigrade); ST: Average July temperature (centigrade). 


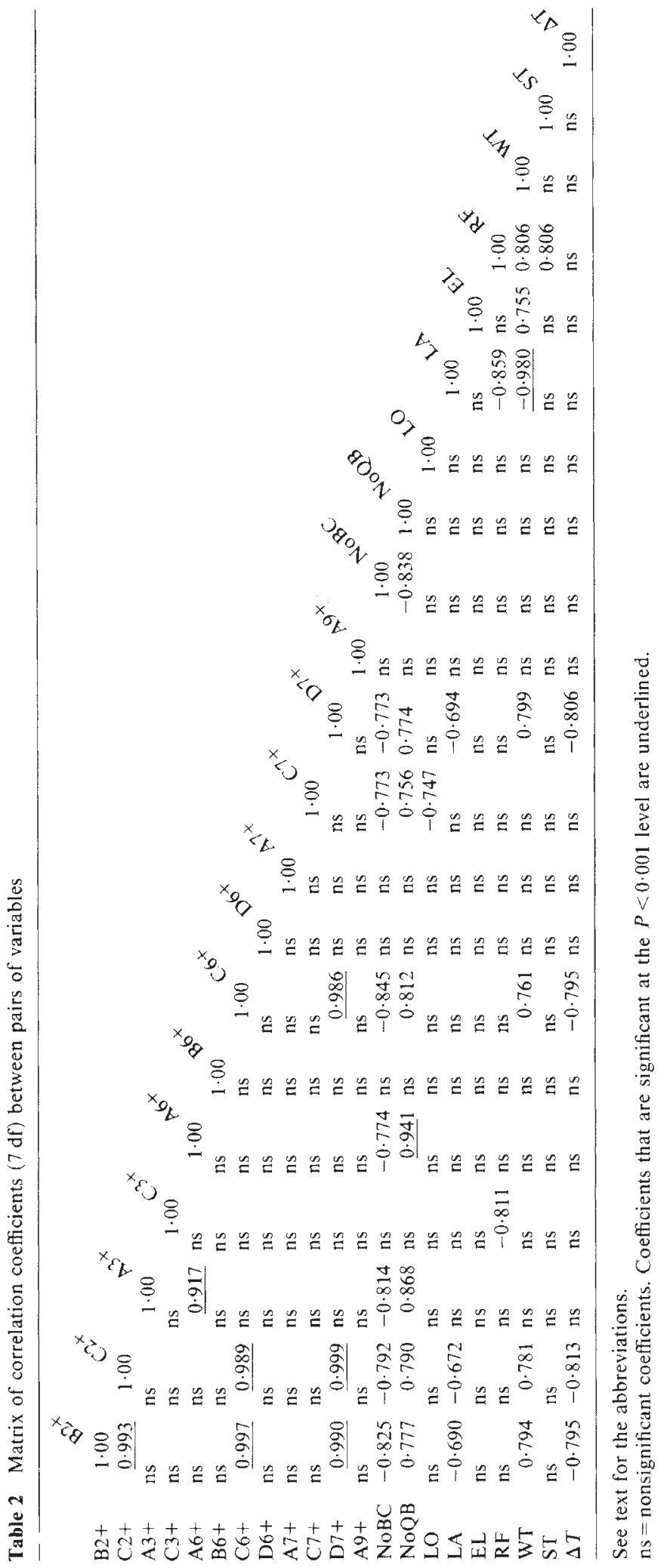


and one including those whose allele frequencies did not; (2) the $F_{\text {st }}$ values in the two groups were compared through Wilcoxon's two-sample test. A significant heterogeneity of $F_{\mathrm{st}}$ values was interpreted as evidence that a pressure associated with the environmental variable of interest has affected the gene frequency variance in one of the groups.

\section{Spatial autocorrelation}

Spatial autocorrelation statistics summarize the relationship between values of the same variable as a function of distances between sampling localities. Inferences on the causal processes are based on the spatial structure detected, and on comparative analysis of several variables (Sokal and Oden, 1978b; Sokal, 1979, 1986).

A $9 \times 9$ matrix of great circle distances between populations was constructed. The pairs of populations were then grouped in four distance classes, defined as follows: 1-50, 50-100, 100-200, and $200-400 \mathrm{~km}$. For each locus and each class, two autocorrelation coefficients, Moran's $I$ and Geary's $c$, were calculated (Sokal and Oden, 1978a; Cliff and Ord, 1981).

Moran's $I$ is defined as

$$
I=\frac{N \sum \sum w_{i j}\left(q_{i}-\bar{q}\right)\left(q_{j}-\bar{q}\right)}{W \sum\left(q_{i}-\bar{q}\right)}
$$

and Geary's $c$ as

$$
c=\frac{(N-1) \sum \sum w_{i j}\left(q_{i}-q_{j}\right)^{2}}{2 W \sum\left(q_{i}-\bar{q}\right)} .
$$

In these formulae, $q_{i}$ and $q_{j}$ are the allele frequencies in the $i$ th and $j$ th sample, respectively; $\bar{q}$ is the mean over the $N$ samples (here: $N=9$ ); $W$ is the number of pairs of samples in the distance class of interest; and $w_{i j}$ is a weighting factor that, in the present study, was equal to 1 for all pairs of samples falling in the distance class considered, and equal to 0 for all other pairs. Standard errors were computed according to Sokal and Oden (1978a) and Ripley (1981).

The values of Moran's I range from 1 (for positive association of allele frequencies at a certain distance), to -1 (for a negative relationship); the expected value is $(N-1)^{-1}$. The corresponding limits of Geary's $c$ are 0 and an undefined positive value; in the absence of any consistent relationship $c$ is expected to be equal to 1 .

The similarity of spatial patterns at various loci was evaluated by constructing a matrix of Manhattan distances between correlograms (Sneath and Sokal, 1973, pp. 125-126), non-significant correlograms excluded. The markers were then grouped according to UPGMA clustering strategy (Sneath and Sokal, 1973, pp. 230-234).

\section{RESULTS}

Table 2 is the matrix of product-moment correlation coefficients between pairs of variables. Forty out of 210 coefficients (main diagonal excluded) were significant at the 0.05 level. Some associations are predictable (e.g., latitude with WT and RF; NoQB with frequency of several bands). A highly significant negative association was apparent between NoBC and NoQB. This relationship was significant also when evaluated at the individual level, by correlating the number of B-chromosomes and Q-bands in 361 plants for which both data were available $(r=-0 \cdot 186, p<0 \cdot 001)$. B2, C2, C6 and D7 were tightly associated (all coefficients $P<0.001$ ); all other correlations between band frequencies were insignificant except one.

The correlation of the $12 \mathrm{Q}$-band frequencies with the seven environmental variables reached significance in one case for $L O$ and RF, three cases for $L A$, and four cases for $W T$ and $\Delta T$. The values of $F_{\text {st }}$ ranged from 0.013 (for B6) to 0.906 (for C6); the median was $0 \cdot 515$. Since the frequency of no bands was associated with EL and ST, only five tests for the heterogeneity of $F_{\text {st }}$ were in fact carried out. Three of them yielded nonsignificant results. Conversely, the four polymorphisms which were associated with $W T$ and $\Delta T$ (namely B2, C2, C6 and D7, in both cases) displayed significantly greater variation than the other eight polymorphisms (Wilcoxon's $U_{\mathrm{s}}=35,4$ and $8 \mathrm{df} ; P<0.025$ in both cases).

Figure 4 is a plot of Moran's $I$ values versus distance, or spatial correlograms. (Geary's $c$ correlograms were almost identical, and are not given). Three distinct modes of geographic variation were apparent among the 14 variables studied. Four polymorphisms (A6, B6, D6 and A7) did not show any significant pattern in space. Four other polymorphisms (B2, C2, C6 and D7) showed significant positive autocorrelation at $50 \mathrm{~km}$, followed by no pattern at larger distances. In a third group of markers, spatial autocorrelation was significantly negative in one of the intermediate distance classes (A3, C7, A9, plus NoBC and NoQB), or in the last one $(\mathrm{C} 3)$. The polymorphisms showing positive short-range autocorrelation also showed similar spatial distributions of their allele frequencies. For all of them, the + allele reached polymorphic frequencies in the outer populations (Vico 

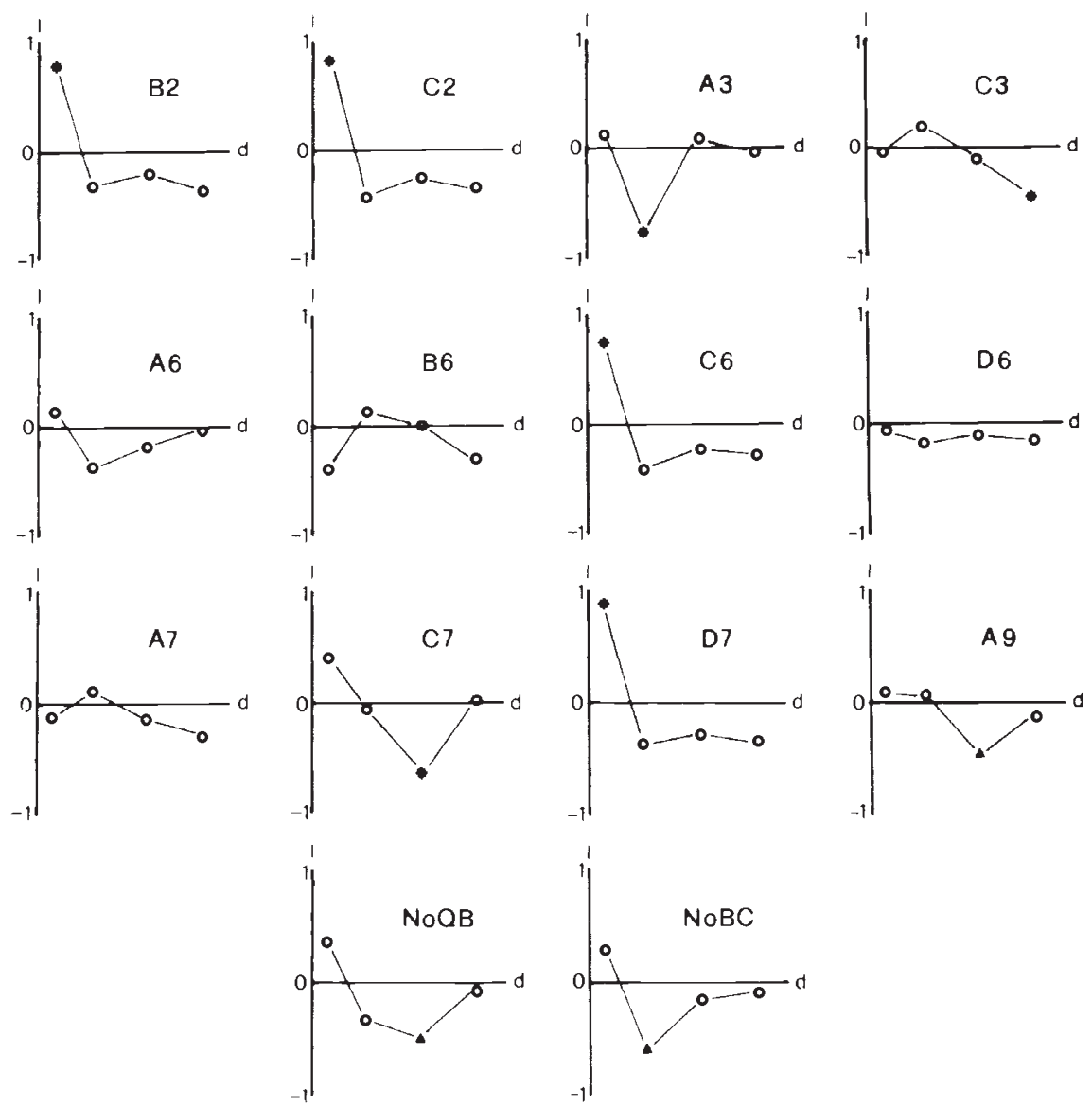

Figure 4 Spatial correlograms of 12 banding polymorphisms, average number of $B$ chromosomes (NoBC), and average number of Q-bands (NoQB) in nine Italian populations of Ornithogalum montanum. Open circles: nonsignificant coefficients; solid triangles: $P<0.05$; stars: $P<0.01$. The upper limits of the four distance classes considered are $50,100,200$, and $400 \mathrm{kilometres}$.

and Aspromonte), but it was not observed in the central part of the Italian range of the species. These loci formed a cluster when the correlograms were grouped by UPGMA strategy (fig. 5), whereas no obvious aggregation of the remaining markers was evident.

\section{DISCUSSION}

The Italian populations of $O$. montanum are geographically differentiated. The karyotypes of the individuals sampled in the central section of the area studied have less $Q$ bands and more $B$ chromosomes than the average. Conversely, some alleles that are absent in these regions ( $\mathrm{B} 2+, \mathrm{C} 2+$, $\mathrm{C} 6+, \mathrm{C} 7+$, and $\mathrm{D} 7+$ ) are common in the northern and southern isolates.
Most karyotype variation appears to be spatially random; the negative autocorrelation occasionally observed at 100 or $200 \mathrm{~km}$ does not suggest any obvious microevolutionary process. However, four markers, namely B2, C2, C6 and D7, vary in a peculiar way. The frequencies of their + alleles are highly associated among them, and all are correlated with $W T$ and $\Delta T$. Their $F_{\text {st }}$ values are significantly higher than those calculated at the other eight loci. Although a single statistical test is no longer considered sufficient for rejecting a neutral or a selective explanation of genetic differentiation (Lewontin, 1985), heterogeneity of $F_{\mathrm{st}}$ values does indicate the characteristics of the habitat to which gene frequency diversity is most significantly associated. Autocorrelation coefficients then confirm that genetic variation at these loci is not random in the 


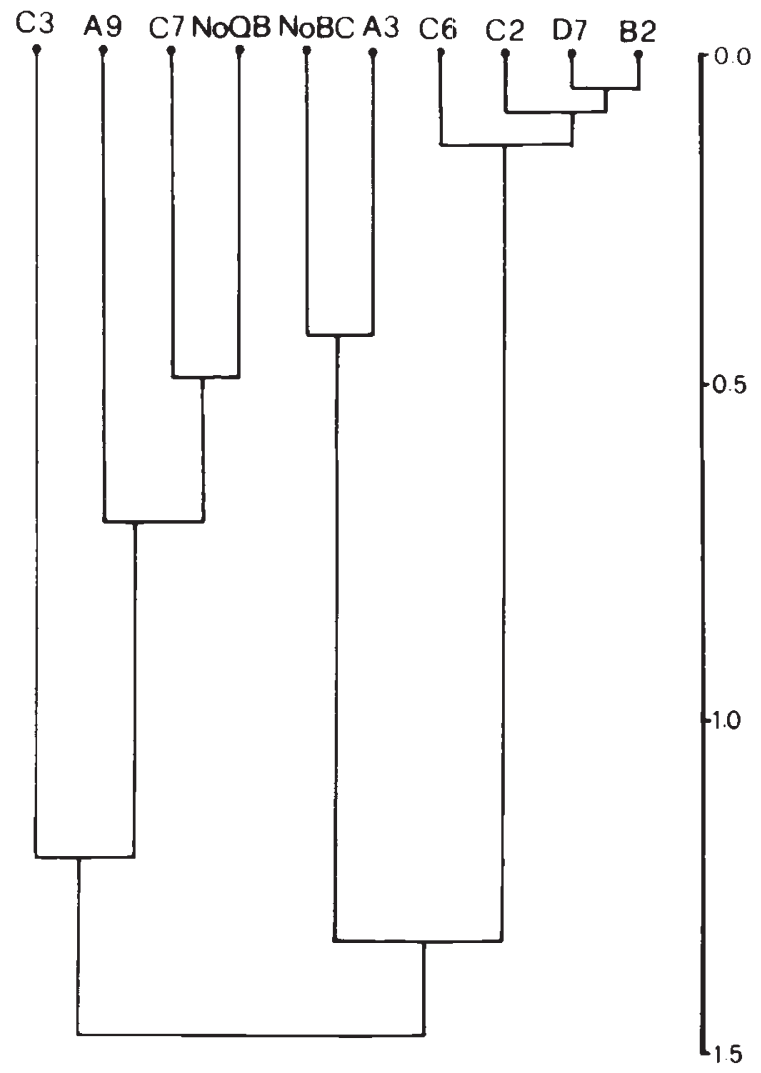

Figure 5 Clustering of the polymorphisms showing spatial structure, based on Manhattan distances between correlograms (Moran's $I$ ).

geographical space (positive short-range autocorrelation).

For the sake of simplicity, we will refer to B2, C2, C6 and D7 as ND-loci (for Nonrandom Distribution of allele frequencies). The karyotype similarity measured at the ND-loci decreases with distance, reaches 0 around $50 \mathrm{~km}$, and then levels off. This profile of the correlograms is expected both under differential selection in an environment where selectively heterogeneous patches are present (Sokal, 1979), and also when genetic differentiation is caused by genetic drift and short-range gene flow, i.e., under isolation by distance (Barbujani, 1987). Under the hypothesis of selection, $50 \mathrm{~km}$ would be a rough estimate of the size of the patches within which a uniform selective pressure has affected karyotype polymorphism (Sokal, 1979); under the hypothesis of isolation by distance, $50 \mathrm{~km}$ would be a measure of the extent to which gene flow has caused neighbouring populations to resemble each other. Fifty kilometres is surely more than a reasonable dispersal distance for Ornithogalum; however, the effects of gene flow tend to accumulate across generations, leading to significant spatial structure on a scale that is large compared to individual dispersal (see e.g., Slatkin, 1978; Piazza and Menozzi, 1983; Barbujani, 1988). In addition, it must be noted that the differences in the putative selective factors, namely winter temperatures and $\Delta T$, among the localities studied, are two degrees at best. Therefore, a hypothetical adaptive mechanism maintaining polymorphism at the ND-loci, or at linked loci, must be sensitive to extremely slight temperature variations.

The answers to the five questions listed in the introduction are in part implicit in this summary of the present study. Geographic variation is nonrandom for four of the twelve loci studied (question 1). This fraction of karyotype variation can be explained by a process of isolation by distance whose range is estimated around $50 \mathrm{~km}$ (question 2). Although distance is a factor, it is not necessarily the only factor maintaining genetic diversity in Italian O. montanum. Apart from selection (to be discussed later), two kinds of evidence suggest that subdivision also plays a role. Firstly, since the + alleles of five markers are absent in the populations of Lungro, Pollino and Sila, the exchange of gametes among these localities and the northern and southern isolates must be scarce, if any. Secondly, the mountain areas where $O$. montanum grows are interrupted by two belts of plains, located south of Vico and north of Aspromonte, respectively, which act as obstacles to gene flow. Therefore, populations separated by large distances may turn out to be genetically unrelated, as they appear to be when spatial distributions of allele frequencies are analysed. As a consequence, the Italian population of $O$. montanum can tentatively be regarded as composed of at least three distinct units (Vico; Pollino + Sila + Lungro; Aspromonte), within which further genetic differentiation is caused by isolation by distance. Only a wider study can show whether this apparently discontinuous variation is to some extent an artifact due to incomplete sampling of the area studied.

Geographical clines have not been observed in the present study; there is no evidence of geographically structured variation of environmental pressures (question 3). Rejecting differential selection as a factor maintaining even a minor fraction of karyotype polymorphism in Italian $O$. montanum, however, leaves unexplained the heterogeneity of $F_{\text {st }}$ values among loci classified according to their relationship with winter temperature and $\Delta T$. Note that winter temperature and 
$\Delta T$ are not correlated, so that $F_{\text {st }}$ is actually heterogeneous in two out of seven tests carried out. Thus, the answer to question 3 is not completely obvious. If the spatial patterns described for the ND-loci are regarded as a consequence of isolation by distance rather than differential selection, the heterogeneity of $F_{\mathrm{st}}$ values observed at these loci, although significant, ought to be attributed to chance.

The number of B-chromosomes is substantially lower when there are many Q-bands in the karyotype (question 4). In general, B chromosomes are detrimental (Jones, 1985), but meiotic drive maintains them despite selection (Shaw, 1983). "Anti B" genes have been described in some species (Nur, unpublished; cited by Jones, 1985). The results of the present study suggest that a function of this type may be carried out by the Quinacrinepositive regions of the genome of $O$. montanum. An intriguing finding is that the negative correlation of numbers of B-chromosomes and Q-bands is higher at the population level, between average numbers $\left(r^{2}=0.702\right)$, than at the single karyotype level $\left(r^{2}=0 \cdot 034\right.$; this coefficient is also significant, because of its 359 degrees of freedom). Therefore, this relationship seems to depend not only on processes affecting the individual plant, but on population structure too. The simplest mechanism accounting for it at the individual level involves alleles in linkage disequilibrium with Q-bands, which apparently counterbalance the accumulation of B-chromosomes; other explanations can be proposed as well. In particular, the opposite possibility (B-chromosomes cause instabilities in the banding patterns) cannot be ruled out in principle. However, this would be an unusual genetic phenomenon, and one which is not supported by direct evidence in this or other studies.

As for question (5), three processes leading to occurrence of the spatial patterns observed in this study can be envisaged: (5.1) mutation + selection; (5.2) mutation + subdivision; (5.3) founder effect + subdivision. In this context, mutation implies that variation arose in the area studied, whereas founder effect is a consequence of differentiation that occurred elsewhere.

Let us first assume that the Q-bands at the ND-loci have originated from mutation in the outer isolates of the Italian range of $O$. montanum, which is where they have been observed in this study. According to process (5.1) they must have spread because of some selective advantage, perhaps related to resistance to low temperatures; according to process (5.2), they must have spread by chance, and their absence in the Central regions must be due mainly to insufficient gene flow. The difficulty with both processes is that they. imply equal and independent chromosome mutation in different populations, which is generally considered highly unlikely. On the other hand, if only unique mutational events are postulated, then the observed pattern of differentiation can have been reached only through extinction of the individuals bearing these bands in the central isolates; this does not seem a parsimonious hypothesis.

Alternatively, process (5.3) explains the spatial patterns at the ND-loci as the result of independent colonization of three areas of Italy by different stocks of O. montanum. Multiple colonization processes by different genotypes have been proposed to account for the extensive variation observed in numerous plant species (see e.g., Shaw et al., 1987). Cullen and Ratter (1967) argued that O. montanum originated in Turkey because of the relative amount of phenotype variation observed, which was maximum in Turkey, and then decreased as a function of distance from there. Italy is at the western limits of the present range of the species, and has apparently been colonized only in its southernmost parts. This suggests that $O$. montanum has spread only recently to Italy, thus indirectly supporting the view that the genotypes of the local populations have diverged elsewhere. Therefore, the karyotype polymorphism observed in the Italian populations of $Q$. montanum seems to reflect the history of these populations, rather than geographically variable evolutionary processes.

Acknowledgements This study was supported by MPI 60 per cent research fund. We are indebted to Professors P. Marchi, G. F. D'Amato, R. Capineri and G. Bianchi for allowing access to their data. Professor A. Piazza kindly provided copy of some of his papers. We thank Professors R. R. Sokal and F. Fontana for discussion, and $\operatorname{Dr}$ A. Farabegoli for skilled technical assistance.

\section{REFERENCES}

ANONymous 1983. Annuario di Statistiche Meteorologiche. ISTAT, Istituto Superiore di Statistica, Roma.

BARBUJANI, G. 1985. A two-step test for the heterogeneity of $F_{\mathrm{st}}$ values at different loci. Hum. Hered., 35, 292-295.

BARBUJANI, G. 1987. Autocorrelation of gene frequencies under isolation by distance. Genetics, 117, 777-782.

BARbUjani, G. 1988. Diversity of some gene frequencies in European and Asian populations. IV. Genetic population structure assessed by the variogram. Ann. Hum. Genet., 52, 215-255.

BRAISHAW, A. D. 1972. Some of the evolutionary consequences of being a plant. Evol. Biol., 5, 25-47. 
CAPINERI, R., D'AMATO, G. F., MARCHI, P., MARAZIA, T. AND MAGGINI, F. 1979. Band pattern polymorphism in Ornithogalum montanum Cyr. ex Ten. (Liliaceae). Atti Ass. Genet. Ital., 24, 83-85.

CAVALLI-SFORZA, L. L. 1966. Population structure and human evolution. Proc. Royal Soc. London, B, 164, 362-379.

ClifF, A. D. AND ORD, J. K. 1981. Spatial Processes. Models and Applications, Pion, London.

CULlen, J. AND RATTER, J. A. 1967. Taxonomic and cytological notes on Turkish Ornithogalum. Notes Royal Bot. Garden, 27, 293-339.

ENDler, J. A. 1977. Geographic Variation, Speciation, and Clines, Princeton University Press, Princeton.

FELSENSTEIN, J. 1982. How can we infer geography and history from gene frequencies? J. Theor. Biol., 96, 9-20.

JONES, R. N. 1985. Are B chromosomes 'selfish'? In CavalierSmith, T. (ed.) The Evolution of (Genome Size, J. Wiley \& Sons, Chichester, pp. 397-425.

JONES, R. N. AND REES, H. 1982. B Chromosomes, Academic Press, New York.

LEWONTIN, R. C. 1985. Population genetics. Ann. Rev. Genet., $19,81-102$.

LEWONTIN, R. C. AND KRAKAUER, J. 1973. Distribution of gene frequency as a test of the theory of the selective neutrality of polymorphisms. Genetics, 74, 175-195.

LEWONTIN, R. C. AND KRAKAUER, J. 1975. Testing the heterogeneity of $F$ values. Genetics, 80, 397-398.

NEI, M., CHAKRAVARTI, A. AND TATENO, Y. 1977. Mean and variance of $F_{\mathrm{st}}$ in a finite number of incompletely isolated populations. Theor. Pop. Biol., 11, 291-306.

NEVO, E., BEILES, A. AND BEN-SHLOMO, R. 1984. The evolutionary significance of genetic diversity: Ecological, demographic and life history correlates. In Mani, G. S. (ed.) Evolutionary Dynamics of Genetic Diversity, Lecture Notes in Biomathematics 53, Springer-Verlag, New York, pp. 13-213.

PIAZZA, A. AND MENOZZI, P. 1983. Geographic variation in human gene frequencies. In Felsenstein, J. (ed.) Numerical Taxonomy, Springer, Berlin, pp. 444-450.

RIPLEY, B. D. 1981. Spatial Statistics, J. Wiley \& Sons, New York.

ROHLF, F. J. AND SCHNELL, G. D. 1971. An investigation of the isolation by distance model. Am. Nat., 105, 295-324.
SHAW, J., MEAGHER, T. R. AND HARLEY, P. 1987. Electrophoretic evidence of reproductive isolation between two varieties of the moss, Climacium americanum. Heredity, 59, 337-343.

SHAw, M. W. 1983. Movement of a cline for supernumerary chromosomes. In Brandam, P. E. and Bennett, M. D. (eds) Kew Chromosome Conference II, George Allen \& Unwin, London, pp. 217-223.

SLATKIN, M. 1978. Spatial patterns in the distributions of polygenic characters. J. Theor. Biol., 70, 213-228.

SNEATH, P. H. A. AND SOKAL, R. R. 1973. Numerical Taxonomy, Freeman, San Francisco.

SOKAL, R. R. 1979. Ecological parameters inferred from spatial correlograms. In Patil, G. P. and Rosenzweig, M. L. (eds) Contemporary Quantitative Ecology and Related Econometrics, International Co-operative Publishing House, Fairland, Maryland, pp. 167-196.

SOKAL, R. R. 1986. Spatial data analysis and historical processes. In Diday, E., Escoufier, Y., Lebart, L., Pages, J., Schektman, Y., Tomassone, R. (eds) Data Analysis and Information, Elsevier, Amsterdam, pp. 29-43.

SOKAL, R. R. AND ODEN, N. L. 1978a. Spatial autocorrelation in biology. 1. Methodology. Biol. J. Linn. Soc., 10, 199-228.

SOKAL, R. R. AND ODEN, N. L. 1978 b. Spatial autocorrelation in biology. 2. Some biological implications and four applications of evolutionary and ecological interest. Biol. J. Linn. Soc. 10, 229-249.

SOKAL, R. R. AND WARTENBERG, D. 1983. A test of spatial autocorrelation analysis using an isolation-by-distance model. Genetics, 105, 219-237.

SULTAN, S. E. 1987. Evolutionary implications of phenotypic plasticity in plants. Evol. Biol., 21, 127-178.

TORNADORE, N. 1986. Gen. Ornithogalum L. (Liliaceae). VII Attuali conoscenze sulla cariologia del genere per il territorio italiano. Giorn. Bot. Ital., 120, Suppl. 2, 178.

WRIGHT, S. 1965. The interpretation of population structure by $F$-statistics with special regard to systems of mating. Evolution, 19, 395-420.

WRIGHT, S. 1969. Evolution and the Genetics of Populations, Vol. 2: The Theory of Gene Frequencies, Chicago University Press, Chicago. 